Published in "Geometriae Dedicata 200(1): 45-59, 2019"

which should be cited to refer to this work.

\title{
On right-angled polygons in hyperbolic space
}

\author{
Edoardo Dotti ${ }^{1}$. Simon T. Drewitz ${ }^{1}$
}

\begin{abstract}
We study oriented right-angled polygons in hyperbolic spaces of arbitrary dimensions, that is, finite sequences $\left(S_{0}, S_{1}, \ldots, S_{p-1}\right)$ of oriented geodesics in the hyperbolic space $\boldsymbol{H}^{n+2}$ such that consecutive sides are orthogonal. It was previously shown by Delgove and Retailleau (Ann Fac Sci Toulouse Math 23(5):1049-1061, 2014. https://doi.org/ 10.5802/afst.1435) that three quaternionic parameters define a right-angled hexagon in the 5-dimensional hyperbolic space. We generalise this method to right-angled polygons with an arbitrary number of sides $p \geq 5$ in a hyperbolic space of arbitrary dimension.
\end{abstract}

Keywords Hyperbolic space $\cdot$ Clifford matrix $\cdot$ Cross ratio $\cdot$ Right-angled polygon $\cdot$ Golden ratio

Mathematics Subject Classification (2000) 51M10 $15 \mathrm{~A} 66 \cdot 51 \mathrm{M} 20$

\section{Introduction}

For $n \geq 0$, let $\boldsymbol{H}^{n+2}$ denote the real hyperbolic $(n+2)$-space. The boundary of this space can be described with Clifford vectors. These are special elements of the Clifford algebra $\mathcal{C}_{n}$, which is the unitary associative algebra generated by $n$ elements $i_{1}, \ldots, i_{n}$ such that $i_{j} i_{l}=$ $-i_{l} i_{j}, i_{l}^{2}=-1$ for $l \neq j$. The group of orientation preserving isometries $\operatorname{Isom}^{+}\left(\boldsymbol{H}^{n+2}\right)$ of the hyperbolic space $\boldsymbol{H}^{n+2}$ can be expressed with Clifford matrices. These are $2 \times 2$ matrices

Edoardo Dotti was partially supported by the Schweizerischer Nationalfonds 200020-156104.

$凶 \quad$ Simon T. Drewitz

simon.drewitz@unifr.ch

Edoardo Dotti

edoardo.dotti@unifr.ch

1 Département de mathématiques, Université de Fribourg, Chemin du Musée 23, 1700 Fribourg, Switzerland 
with coefficients in the multiplicative group generated by Clifford vectors and with Ahlfors determinant 1 .

In this context, we describe hyperbolic right-angled polygons for which we mean rightangled closed edge paths in $n+2$ dimensions. We show how to construct a hyperbolic right-angled polygon $\Pi_{p}$ of $p$ sides, $p>4$, by prescribing a parameter set consisting of $p-3$ Clifford vectors in $\partial \boldsymbol{H}^{n+2}$. Such a construction is achieved in an arbitrary dimension. No connection between the dimension of the space and the number of sides of the polygon is required.

Similar objects have already been studied in dimension 2 and 3 by Thurston [8] and by Fenchel [9], who studied right-angled hexagons. Costa and Martínez [5] studied right-angled polygons with an arbitrary number of sides in the hyperbolic plane. More recently Delgove and Retailleau [7] classified right-angled hexagons in $\boldsymbol{H}^{5}$. In their work, $2 \times 2$ quaternionic matrices having Dieudonné determinant 1 are used in order to describe the direct isometries of $\boldsymbol{H}^{5}$. While this approach based on quaternions is very convenient, it can not be extended to arbitrary dimensions. By using Clifford matrices instead, we are able to generalise the construction to any dimension. Particularly, $2 \times 2$ quaternionic Clifford matrices are used to describe direct isometries of $\boldsymbol{H}^{4}$.

In the first section we develop more precisely the connection between hyperbolic space and the Clifford algebra. Then we discuss the role of the cross ratio for Clifford vectors and its geometrical interpretation. Our main result, the algorithmic construction of $\Pi_{p}$, is presented in the second section. In the last part we treat the case when the convex hull of the $p$ vertices of the polygon $\Pi_{p}$ give rise to a hyperbolic $(p-1)$-simplex. A necessary condition for its realisation is stated. As a conclusion we discuss in more details a special case in 4 dimensions, supposing that all the edges of the edge path have equal length. By exploiting the work of Dekster and Wilker [6] we explicitly state a necessary and sufficient condition for realisability depending on such a side length. Surprisingly, it turns out that the side length must be related to the golden ratio $\gamma=\frac{1+\sqrt{5}}{2}$.

\section{The real Clifford algebra and hyperbolic space}

In this section we present the notion of Clifford algebra and its relation to isometries of hyperbolic space. For a more complete description we refer to the works of Ahlfors [1,2], Vahlen [13] and Waterman [14] (see also [11, Section 7]).

\subsection{The real Clifford algebra $\mathcal{C}_{n}$}

Consider the real Clifford algebra $\mathcal{C}_{n}$ generated by $i_{1}, \ldots, i_{n}$, that is

$$
\left.\mathcal{C}_{n}=\left\langle i_{1}, \ldots, i_{n}\right| i_{j} i_{l}=-i_{l} i_{j}, i_{l}^{2}=-1 \text { for } l \neq j\right\rangle,
$$

which is a unitary associative real algebra. Every element $x$ of the algebra $\mathcal{C}_{n}$ can be uniquely written as $x=\sum x_{I} I$, where $x_{I} \in \mathbb{R}$ and the sum is taken over all the products $I=i_{k_{1}} \cdots i_{k_{m}}$, with $1 \leq k_{1}<\cdots<k_{m} \leq n$ and $1 \leq m \leq n$. Here the empty product $I_{0}$ is included and identified with $i_{0}:=1$. Hence $\mathcal{C}_{n}$ is a $2^{n}$-dimensional real vector space. In particular we can identify $\mathcal{C}_{0}$ with $\mathbb{R}, \mathcal{C}_{1}$ with $\mathbb{C}$ and $\mathcal{C}_{2}$ with $\mathbb{H}$, the Hamiltonian quaternions. To each element $x=\sum x_{I} I$ we associate a norm as given by $|x|^{2}=\sum x_{I}^{2}$, inducing a Euclidean structure on $\mathcal{C}_{n}$. Denote with $\Re(x)$ the coefficient $x_{0}$, called the real part of $x$, while $\Im(x)=x-\Re(x)$ is called the non-real part of $x$. If $\Re(x)=0$ we will refer to $x$ as a pure element of $\mathcal{C}_{n}$. 
On $\mathcal{C}_{n}$ there are three well-known involutions. Let $x \in \mathcal{C}_{n}, x=\sum x_{I} I$. Then:

(i) $x^{*}=\sum x_{I} I^{*}$, where $I^{*}$ is obtained from $I=i_{k_{1}} \cdots i_{k_{m}}$ by reversing the order of the factors, that is $I^{*}=i_{k_{m}} \cdots i_{k_{1}}$;

(ii) $x^{\prime}=\sum x_{I} I^{\prime}$, where $I^{\prime}$ is obtained from $I=i_{k_{1}} \cdots i_{k_{m}}$ by replacing each factor $i_{k}$ with $-i_{k}$, that is $I^{\prime}=\left(-i_{k_{1}}\right) \cdots\left(-i_{k_{m}}\right)=(-1)^{m} I$

(iii) $\bar{x}=\left(x^{*}\right)^{\prime}=\left(x^{\prime}\right)^{*}$.

The involutions (i) and (iii) are anti-automorphisms, while the involution (ii) is an automorphism.

Of particular interest are Clifford elements of the form $x=x_{0}+x_{1} i_{1}+\cdots+x_{n} i_{n}$, called Clifford vectors. The set

$$
\mathbb{V}^{n+1}=\left\{x_{0}+x_{1} i_{1}+\cdots+x_{n} i_{n} \mid x_{0}, \ldots, x_{n} \in \mathbb{R}\right\}
$$

of all Clifford vectors is an $(n+1)$-dimensional real vector space, naturally isomorphic to the Euclidean space $\mathbb{R}^{n+1}$. Notice that for an element $x \in \mathbb{V}^{n+1}$ we have $x^{*}=x$ and hence $\bar{x}=x^{\prime}$ as well as $x+\bar{x}=2 \Re(x)$ and $x \bar{x}=\bar{x} x=|x|^{2}$. Moreover every non-zero vector $x$ has an inverse given by $x^{-1}=\frac{\bar{x}}{|x|^{2}}$. Hence finite products of non-zero vectors are invertible and they form the so-called Clifford group $\Gamma_{n}$. Observe that we have $\Gamma_{n}=\mathcal{C}_{n} \backslash\{0\}$ only for $n \in\{0,1,2\}$.

\subsection{Square root of a Clifford vector}

Next we introduce the notion of the square root of a Clifford vector. It will be a generalisation of the square root of quaternions (see [10] for example) in the following way:

Proposition 1 Let $y \in \mathbb{V}^{n+1} \backslash\{0\}$ be a Clifford vector. If $y \notin \mathbb{R}_{<0}$, then there exist exactly two elements $x_{1}, x_{2} \in \mathbb{V}^{n+1}$ such that $x_{1}^{2}=x_{2}^{2}=y ; x_{1}$ and $x_{2}$ are both called a square root of $y$. If $y \in \mathbb{R}_{<0}$, we have the three following situations depending on $n$ :

- If $n=0$, then there is no element $x \in \mathbb{V}^{1}$ such that $x^{2}=y$,

- If $n=1$, then there are exactly two elements $x_{1}, x_{2} \in \mathbb{V}^{2}$ such that $x_{1}^{2}=x_{2}^{2}=y$,

- If $n \geq 2$, then there are uncountably many square roots of $y$.

Proof Suppose that $x^{2}=y$, with $x, y \in \mathbb{V}^{n+1} \backslash\{0\}$. Then $\bar{x}^{2}=\bar{y}$ and $|x|^{2}=|y|$. We have the following two equations:

$$
\begin{aligned}
x(\bar{x}+x) & =x \bar{x}+x^{2}=|y|+y, \\
(x+\bar{x})^{2} & =x^{2}+2 x \bar{x}+\bar{x}^{2}=y+2|y|+\bar{y}=2(\Re(y)+|y|) .
\end{aligned}
$$

Observe that the term $2(\Re(y)+|y|) \geq 0$.

Now let $y \notin \mathbb{R}_{<0}$, then we have $\Re(y)+|y|>0$, and the element

$$
x:=\frac{|y|+y}{\sqrt{2(\Re(y)+|y|)}} \in \mathbb{V}^{n+1}
$$

satisfies $x^{2}=y$. Indeed,

$$
x^{2}=\frac{|y|^{2}+2|y| y+y^{2}}{2 \Re(y)+2|y|}=\frac{(\bar{y}+2|y|+y) y}{2 \Re(y)+2|y|}=y .
$$

Notice that in the special case if $y \in \mathbb{R}_{>0}$, the identity (3) yields $x= \pm \sqrt{y}$ as desired. For $y \notin \mathbb{R}$ the square roots of $y$ have to lie in the plane spanned by 1 and $y$ which is isomorphic 
to $\mathbb{C}$, ensuring the non-existence of more than two roots. By abuse of notation the square root $x$ of $y$ is denoted by $\sqrt{y}:=x$.

Let $y \in \mathbb{R}_{<0}$. For $n=0$ or 1 the assertion is trivial. Let $n \geq 2$. We can write $y=-z^{2}$ for some $z \in \mathbb{R}_{>0}$. In this case consider $x:=z \cdot u$ where $u$ is a pure Clifford vector with norm 1. In general for any pure Clifford vector we have

$$
0=(u+\bar{u}) u=u \bar{u}+u^{2}=|u|^{2}+u^{2},
$$

which implies $u^{2}=-|u|^{2}$. Hence $x^{2}=z^{2} u^{2}=-z^{2}|u|^{2}=-z^{2}$.

Remark 1 Notice that Proposition 1 remains true for $y \in \Gamma_{2}=\mathbb{H} \backslash\{0\}$ since $y+\bar{y}=2 \Re(y)$ still holds. However, it does not hold for a general element of $\mathcal{C}_{n}$ or even $\Gamma_{n}, n \geq 3$. Indeed, for an arbitrary $y \in \Gamma_{n}$ one has $y+\bar{y} \neq 2 \Re(y)$. For example let $y=i_{1} i_{2} i_{3} \in \Gamma_{n}, n \geq 3$. Then $y+\bar{y}=2 i_{1} i_{2} i_{3}$. Hence Eq. (2) does not hold.

Remark 2 For the square root $\sqrt{y}$ of a Clifford vector $y \in \mathbb{V}^{n+1} \backslash \mathbb{R}_{\leq 0}$ we have:

- For all positive $\mu \in \mathbb{R}_{>0}, \sqrt{\mu y}=\sqrt{\mu} \sqrt{y}$,

- For the inverse $\sqrt{y^{-1}}=\sqrt{y}^{-1}=\frac{1}{|y|} \sqrt{\bar{y}}$.

- The square root of $-y$ can be found by a rotation of $90^{\circ}: \sqrt{-y}=i \sqrt{y}$ for some pure Clifford vector $i$ with $i^{2}=-1$. This also holds for negative $y \in \mathbb{R}_{<0}$.

\subsection{Clifford matrices and hyperbolic isometries}

We now take a look at matrices having entries in the extended Clifford group $\Gamma_{n} \cup\{0\}$. These matrices will be used to explicitly represent direct isometries of the hyperbolic space $\boldsymbol{H}^{n+2}$ (see for example [14] and [11, Section 7]).

A Clifford matrix is a $2 \times 2$ matrix $A=\left(\begin{array}{ll}a & b \\ c & d\end{array}\right)$ with

$$
a, b, c, d \in \Gamma_{n} \cup\{0\}, \quad a b^{*}, c d^{*}, c^{*} a, d^{*} b \in \mathbb{V}^{n+1}, \quad a d^{*}-b c^{*} \in \mathbb{R} \backslash\{0\},
$$

where $a d^{*}-b c^{*}$ is the Ahlfors determinant of $A$. Denote the set of such matrices by $\operatorname{GL}\left(2, \mathcal{C}_{n}\right)$. By a result of Vahlen and Maass [2, p. 221] the set

$$
\operatorname{SL}\left(2, \mathcal{C}_{n}\right)=\left\{A=\left(\begin{array}{ll}
a & b \\
c & d
\end{array}\right) \in \operatorname{GL}\left(2, \mathcal{C}_{n}\right) \mid a d^{*}-b c^{*}=1\right\}
$$

of Clifford matrices with Ahlfors determinant 1 is a multiplicative group.

Each element $T=\left(\begin{array}{ll}a & b \\ c & d\end{array}\right) \in \operatorname{SL}\left(2, \mathcal{C}_{n}\right)$ has the inverse matrix $T^{-1}=\left(\begin{array}{cc}d^{*} & -b^{*} \\ -c^{*} & a^{*}\end{array}\right)$.

Furthermore $\operatorname{SL}\left(2, \mathcal{C}_{n}\right)$ is generated by the matrices

$$
\left(\begin{array}{ll}
1 & t \\
0 & 1
\end{array}\right), \quad\left(\begin{array}{cc}
0 & -1 \\
1 & 0
\end{array}\right), \quad\left(\begin{array}{cc}
a & 0 \\
0 & a^{*-1}
\end{array}\right)
$$

where $t \in \mathbb{V}^{n+1}$ and $a \in \Gamma_{n}$ (see for example [11, Section 7]).

The group $\operatorname{SL}\left(2, \mathcal{C}_{n}\right)$ plays an important role in our investigation since it is closely related to the group of orientation preserving isometries of the hyperbolic $(n+2)$-space realised in the upper half-space according to

$$
\begin{aligned}
\boldsymbol{H}^{n+2} & =\left\{x=\left(x_{0}, x_{1}, \ldots, x_{n+1}\right) \in \mathbb{R}^{n+2} \mid x_{n+1}>0\right\} \\
& \cong \mathbb{V}^{n+1} \times \mathbb{R}_{>0} .
\end{aligned}
$$


The compactification $\overline{\boldsymbol{H}^{n+2}}$ is given by the union of $\boldsymbol{H}^{n+2}$ with the boundary set $\partial \boldsymbol{H}^{n+2}=$ $\mathbb{V}^{n+1} \cup\{\infty\}$ of points at infinity of $\boldsymbol{H}^{n+2}$.

Consider the projective group

$$
\operatorname{PSL}\left(2, \mathcal{C}_{n}\right)=\operatorname{SL}\left(2, \mathcal{C}_{n}\right) /\{ \pm I\}
$$

It is known that this group acts bijectively on $\mathbb{V}^{n+1} \cup\{\infty\}$ by

$$
T(x)=(a x+b)(c x+d)^{-1}
$$

with $T\left(-c^{-1} d\right)=\infty, T(\infty)=a c^{-1}$ if $c \neq 0$, and $T(\infty)=\infty$ otherwise. By Poincaré extension, the action (5) can be extended to the upper half-space $\boldsymbol{H}^{n+2}$. In this way we obtain an isomorphism between $\operatorname{PSL}\left(2, \mathcal{C}_{n}\right)$ and the group $\mathrm{Möb}^{+}(n+1)$ of orientation preserving Möbius transformations of $\mathbb{V}^{n+1} \cup\{\infty\}$ (see [4,14]). Since the group Isom ${ }^{+}\left(\boldsymbol{H}^{n+2}\right)$ of orientation preserving isometries of $\boldsymbol{H}^{n+2}$ is isomorphic to $\mathrm{Möb}^{+}(n+1)$, we get the following identification:

$$
\operatorname{Isom}^{+}\left(\boldsymbol{H}^{n+2}\right) \cong \mathrm{Möb}^{+}(n+1) \cong \operatorname{PSL}\left(2, \mathcal{C}_{n}\right) .
$$

Therefore any direct isometry of $\boldsymbol{H}^{n+2}$ can be represented by a Clifford matrix in $\operatorname{PSL}\left(2, \mathcal{C}_{n}\right)$.

Finally, we remark that Möbius transformations act triply transitively on $\mathbb{V}^{n+1} \cup\{\infty\}$ (see [15, Section 6], for example). That is, given two triplets $\left\{x_{1}, x_{2}, x_{3}\right\}$ and $\left\{x_{1}^{\prime}, x_{2}^{\prime}, x_{3}^{\prime}\right\}$ of distinct points in the boundary, there always exists a transformation $T \in \operatorname{Möb}(n+1)$ with $T\left(x_{i}\right)=x_{i}^{\prime}$. For $n=0$ this map is unique and for $n=1$ it is unique if one demands that it preserves the orientation. In higher dimensions this map is not unique anymore.

\subsection{The cross ratio}

As in the classical case, we shall use the cross ratio to study configurations of points in $\mathbb{V}^{n+1} \cup\{\infty\}$.

Definition 1 Let $x, y, z, w$ be four pairwise different Clifford vectors in $\mathbb{V}^{n+1}$. Then

$$
[x, y, z, w]:=(x-z)(x-w)^{-1}(y-w)(y-z)^{-1} \in \Gamma_{n} \backslash\{0\}
$$

is called the cross ratio of $x, y, z$ and $w$.

We extend the definition (7) by continuity to $\mathbb{V}^{n+1} \cup\{\infty\}$, allowing $x, y$ or $w$ to be $\infty$, by

$$
[\infty, y, z, w]=(y-w)(y-z)^{-1} \text { for } x=\infty,
$$

and similarly for $y=\infty$ and $w=\infty$. Moreover in an analogous way we put

$$
[x, y, \infty, w]=(x-w)^{-1}(y-w) .
$$

The cross ratio satisfies the following transformation behaviour (see [4, Lemma 6.2]):

$$
[T(x), T(y), T(z), T(w)]=(c z+d)^{*-1}[x, y, z, w](c z+d)^{*},
$$

for all $T=\left(\begin{array}{ll}a & b \\ c & d\end{array}\right) \in \operatorname{SL}\left(2, \mathcal{C}_{n}\right)$.

Hence, the real part and the norm of the cross ratio $[x, y, z, w]$ of four vectors are invariant under the action of $T$. However, the cross ratio itself is not an invariant.

We specialise the cross ratio in the following way: consider two oriented geodesics $s, t$ in $\boldsymbol{H}^{n+2}$ whose endpoints $s^{-}, s^{+}$and $t^{-}, t^{+}$are four distinct points in $\mathbb{V}^{n+1} \cup\{\infty\}$. 
Definition 2 The cross ratio $\Delta(s, t)$ of $s$ and $t$ is defined by

$$
\Delta(s, t):=\left[s^{-}, s^{+}, t^{-}, t^{+}\right] .
$$

Lemma 1 Let $s$ and $t$ be two geodesics as above. If $s$ and t intersect then $\Delta(s, t)=\Delta(t, s)$. If $s$ and $t$ are disjoint, then $\Delta(s, t)=\Delta(t, s)$ if one of the endpoints is $\infty$ or if the cross ratios are real, otherwise the two cross ratios are conjugate.

Proof Assuming one of the endpoints to be infinity, let $s=(x, \infty)$ with $x \in \mathbb{V}^{n+1}$. We can apply a translation $\left(\begin{array}{cc}1 & -x \\ 0 & 1\end{array}\right)$ such that $s$ is mapped to $(0, \infty)$. By (9), any translation leaves the cross ratio unchanged. Using (8) it is easy to see that $\Delta(s, t)=\Delta(t, s)$.

Let now $s$ and $t$ be two arbitrary geodesics with no endpoint at infinity. We know that we can always find an isometry $T=\left(\begin{array}{ll}a & b \\ c & d\end{array}\right) \in \operatorname{SL}\left(2, \mathcal{C}_{n}\right)$ mapping the two endpoints of one of the geodesics to 0 and $\infty$. Using (9) and what we have just discussed above we get

$$
\begin{aligned}
\left(c t^{-}+d\right)^{*-1}\left[s^{-}, s^{+}, t^{-}, t^{+}\right]\left(c t^{-}+d\right)^{*} & =\left[T\left(s^{-}\right), T\left(s^{+}\right), T\left(t^{-}\right), T\left(t^{+}\right)\right] \\
& =\left[T\left(t^{-}\right), T\left(t^{+}\right), T\left(s^{-}\right), T\left(s^{+}\right)\right] \\
& =\left(c s^{-}+d\right)^{*-1}\left[t^{-}, t^{+}, s^{-}, s^{+}\right]\left(c s^{-}+d\right)^{*} .
\end{aligned}
$$

Hence the two cross ratios $\Delta(s, t)$ and $\Delta(t, s)$ are conjugate. This implies that if the cross ratios are real, then the equality $\Delta(s, t)=\Delta(t, s)$ holds. In particular, if two geodesics intersect, then $\Delta(s, t)=\Delta(t, s)$ by Proposition 2 below.

Now consider three geodesics $r, s$ and $t$ in $\boldsymbol{H}^{n+2}$ with pairwise different endpoints $r^{-}, r^{+}, s^{-}, s^{+}$and $t^{-}, t^{+}$in $\mathbb{V}^{n-1} \cup\{\infty\}$.

Definition 3 The quantity

$$
\Delta(r, s, t):=\left[s^{+}, s^{-}, r^{+}, t^{+}\right]
$$

is called the double bridge cross ratio of $(r, s, t)$.

Definition 4 The ordered triple $(r, s, t)$ is called a double bridge if $s$ is orthogonal to $r$ and $t$ such that $r \neq t$. If $|\Delta(r, s, t)|>1$, then the intersections $r \cap s$ and $s \cap t$ do not coincide and we call the double bridge properly oriented.

Consider a properly oriented double bridge $(r, s, t)$. The norm of $\Delta(r, s, t)$ encodes the hyperbolic length of the geodesic segment $[r, t]$ on $s$ between $r$ and $t$. Indeed, assume w.l.o.g. that the endpoints of $s$ in the double bridge $(r, s, t)$ are $s^{-}=0$ and $s^{+}=\infty$ (see Fig. 1). The hyperbolic distance $\delta$ of two points $p, q \in s$ in $\boldsymbol{H}^{n+2}$ with $p_{n+2}>q_{n+2}$ is equal to (see [3, p. 131])

$$
\delta=\log \left(\frac{p_{n+2}}{q_{n+2}}\right) .
$$

On the other hand, by (7) we get

$$
|\Delta(r, s, t)|=\left|\left[\infty, 0, r^{+}, t^{+}\right]\right|=\frac{\left|t^{+}\right|}{\left|r^{+}\right|} .
$$

If we take $p=s \cap t$ and $q=r \cap s$, we conclude that $\delta=\log (|\Delta(r, s, t)|)$.

The following results will be of importance: 
Fig. 1 Double bridge

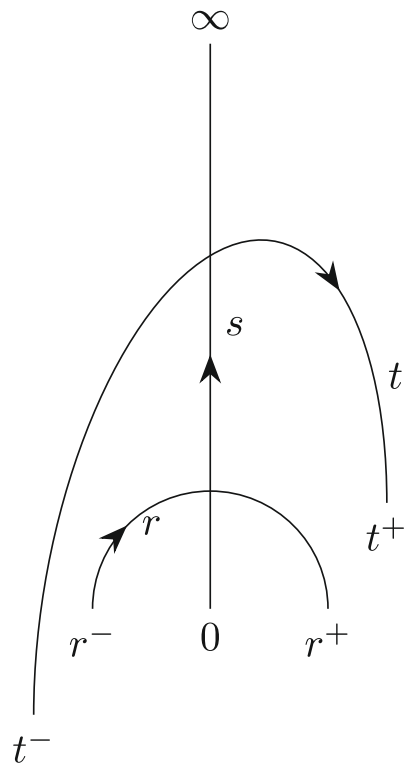

Proposition 2 Two hyperbolic geodesics $s$ and $t$ intersect if and only if their cross ratio $\Delta(s, t) \in \mathbb{R}_{<0}$. Furthermore $s$ and $t$ are perpendicular if and only if $\Delta(s, t)=-1$.

Proof Since hyperbolic isometries act triply transitively, there is an isometry represented by $A \in \operatorname{SL}\left(2, \mathcal{C}_{n}\right)$ mapping $s$ and $t$ into $(0, \infty)$ and $(1, x), x \in \mathbb{V}^{n+1}$. Then, by (7) and (8), the cross ratio of $A(s)$ and $A(t)$ equals $\Delta(A(s), A(t))=[0, \infty, 1, x]=x^{-1}$, and the assertions follow for $A(s)$ and $A(t)$. Moreover, by (9), a real cross ratio stays invariant under isometry.

Proposition 3 Let $s=(0, \infty)$ and $t=(1, y)$ with $y \neq 0, \infty$ be two disjoint geodesics in $\boldsymbol{H}^{n+2}$. Then the common perpendicularl is $(-\sqrt{y}, \sqrt{y})$. This perpendicular is unique up to orientation.

Proof Let $l=(z, w)$ denote the common perpendicular between $s$ and $t$. By Proposition 2 and by (8), we get

$$
\Delta(s, l)=[0, \infty, z, w]=-1 .
$$

and

$$
\Delta(t, l)=[1, y, z, w]=-1 .
$$

Equation (12) yields $z=-w$. The Eq. (13) states that

$$
(1-z)(1+z)^{-1}=-(y-z)(y+z)^{-1} .
$$

It is easy to see that $(1-z)(1+z)^{-1}=(1+z)^{-1}(1-z)$, so that

$$
(1-z)(y+z)=-(1+z)(y-z) .
$$

By expanding the above equation we obtain $y=z^{2}$. Notice that by construction, since $s$ and $t$ are disjoint, we have $y \notin \mathbb{R}_{<0}$. Hence, by applying Proposition 1, the result follows for $l=( \pm \sqrt{y}, \mp \sqrt{y})$. 
Fig. 2 Standard configuration double bridge

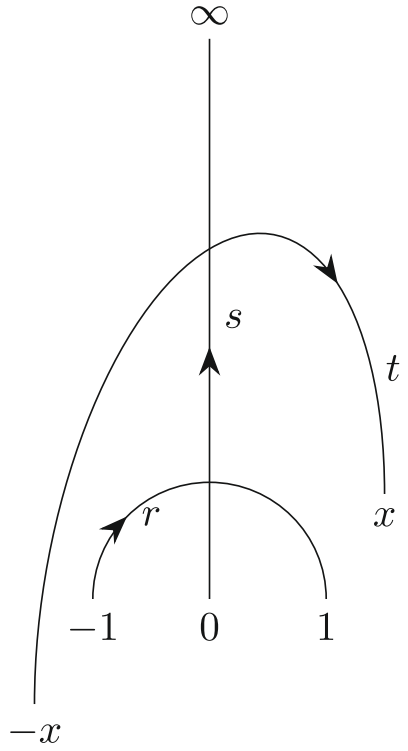

\section{The main theorem}

\subsection{Preliminaries}

Our aim is to construct oriented right-angled polygons in hyperbolic space from a minimal number of prescribed parameters.

Definition 5 An oriented right-angled polygon with $p$ sides in $\boldsymbol{H}^{n+2}$ (or $p$-gon for short), $n \geq 0$, is a $p$-tuple of oriented geodesics $\left(S_{0}, S_{1}, \ldots, S_{p-1}\right)$ with $S_{i-1} \neq S_{i+1}$ for $i(\bmod p)$ and such that $S_{i}$ is orthogonal to $S_{i+1}$ for $0 \leq i \leq p-2$ and $S_{p-1}$ is orthogonal to $S_{0}$.

We usually denote it by $\Pi_{p}$.

We call such a $p$-gon $\Pi_{p}$ non-degenerate if consecutive intersections do not coincide (that is $S_{i-1} \cap S_{i} \neq S_{i} \cap S_{i+1}$ for $\left.i(\bmod p)\right)$ and the double bridges $\left(S_{i-1}, S_{i}, S_{i+1}\right), i(\bmod p)$, are properly oriented.

We can take $p \geq 5$ since the simplest case of a right-angled polygon is the pentagon. There cannot be a hyperbolic rectangle since the common perpendicular of two geodesics $S_{0}$ and $S_{2}$ is unique. Hence if there was a hyperbolic rectangle $\left(S_{0}, S_{1}, S_{2}, S_{3}\right)$, two geodesics would have to be identical.

Note that it is no restriction to only consider $p$-gons in $\boldsymbol{H}^{p-1}$ since the convex hull of $p$ geodesics can at most have dimension $p-1$. Hence, we will always refer to this case.

Recall that the one-point compactified vector space $\mathbb{V}^{p-2} \cup\{\infty\}$ forms the boundary of hyperbolic $(p-1)$-space

$$
\boldsymbol{H}^{p-1}=\left\{(x, y) \in \mathbb{V}^{p-2} \times \mathbb{R}_{>0}\right\} .
$$

Consider the standard configuration double bridge $(r, s, t)$ similar to Sect. 2.4 with $r=$ $(-1,1), s=(0, \infty)$ and $t=(-x, x)$ for $x \in \mathbb{V}^{p-2} \backslash\{-1,0,1\}$ (see Fig. 2).

A small computation shows that the double bridge cross ratio is given by

$$
\Delta((-1,1),(0, \infty),(-x, x))=[\infty, 0,1, x]=x .
$$


If conversely the first two geodesics of this double bridge and a desired double bridge cross ratio $q$ are given, one can construct the third geodesic as $(-q, q)$. In the general case this is not easy since the Clifford vectors do not commute. In view of (15) we shall start with the configuration given by the geodesics $(-1,1),(0, \infty)$ and $(-x, x)$. If the double bridges are supposed to be properly oriented, this poses the immediate restriction $|x|>1$.

To construct more geodesics we will have to apply certain isometries to achieve this configuration from a general double bridge. These isometries depend on the double bridge cross ratios in the right-angled polygon $\Pi_{p}$ they are part of.

Definition 6 For a set of given Clifford vectors $\left\{q_{1}, \ldots, q_{p-3}\right\} \subset \mathbb{V}^{p-2} \backslash\{0\}$ define the isometries $\phi_{i}$ of upper half-space by the following Möbius transformations:

$$
\phi_{i}: x \mapsto{\sqrt{-2 q_{i}}}^{-1}\left(x+q_{i}\right)\left(x-q_{i}\right)^{-1} \sqrt{-2 q_{i}}, \quad 1 \leq i \leq p-3 .
$$

If $q_{i} \in \mathbb{R}_{>0}$, choose $\sqrt{-2 q_{i}}:=\sqrt{2 q_{i}} i_{1}$.

Let $\Phi_{i}$ be the concatenation $\Phi_{i}:=\phi_{i} \circ \phi_{i-1} \circ \cdots \circ \phi_{1}$.

Note that the isometries $\phi_{i}$ carry the two geodesics $(0, \infty)$ and $\left(-q_{i}, q_{i}\right)$ into the geodesics $(-1,1)$ and $(0, \infty)$ of a double bridge in the aforementioned setting. However, these isometries are not uniquely defined by this property. We will always apply these $\phi_{i}$ if we need an isometry which maps given geodesics to specific other geodesics in a polygon $\Pi_{p}$.

The Clifford matrix corresponding to $\phi_{i}$ is

$$
\left(\begin{array}{cc}
{\sqrt{-2 q_{i}}}^{-1} & q_{i}{\sqrt{-2 q_{i}}}^{-1} \\
{\sqrt{-2 q_{i}}}^{-1} & -q_{i} \sqrt{-2 q_{i}}-1
\end{array}\right) .
$$

The inverse $\phi_{i}^{-1}(x)=\sqrt{-q_{i}}(1+x)(1-x)^{-1} \sqrt{-q_{i}}$ is represented by the matrix

$$
\left(\begin{array}{cc}
q_{i}{\sqrt{-2 q_{i}}}^{-1} & q_{i} \sqrt{-2 q_{i}}-1 \\
\sqrt{-2 q_{i}}-1 & -\sqrt{-2 q_{i}}-1
\end{array}\right) .
$$

Repeatedly applying these isometries to geodesics in a $\Pi_{p}$ enables us to standardise the cross ratio of a double bridge in a $p$-gon and eliminate the problem of the cross ratio not being invariant under isometries (Fig. 3).

\subsection{The theorem}

Definition 7 Let $\left(S_{0}, \ldots, S_{p-1}\right)$ be a right-angled $p$-gon. Define the gauged double bridge cross ratios $\tilde{\Delta}_{i}$ for $i=1, \ldots, p-3$ by the following recursive definition:

$$
\begin{aligned}
\tilde{\Delta}_{1} & :=\Delta\left(S_{0}, S_{1}, S_{2}\right), \\
\tilde{\Delta}_{i+1} & :=\Delta\left(\Phi_{i}\left(S_{i}\right), \Phi_{i}\left(S_{i+1}\right), \Phi_{i}\left(S_{i+2}\right)\right) .
\end{aligned}
$$

The Clifford vectors $q_{i}$ which are needed to define the maps $\Phi_{i}$ are calculated along the way as

$$
q_{i}=\tilde{\Delta}_{i}
$$

These gauged double bridge cross ratios will be the parameters describing the nondegenerate right-angled $p$-gons in $\boldsymbol{H}^{p-1}$ in the Theorem 1 below. Hence consider the set

$$
\mathcal{P}_{p}:=\left\{\left(q_{1}, \ldots, q_{p-3}\right)\left|q_{i} \in \mathbb{V}^{p-2},\right| q_{i} \mid>1,1 \leq i \leq p-3\right\}
$$




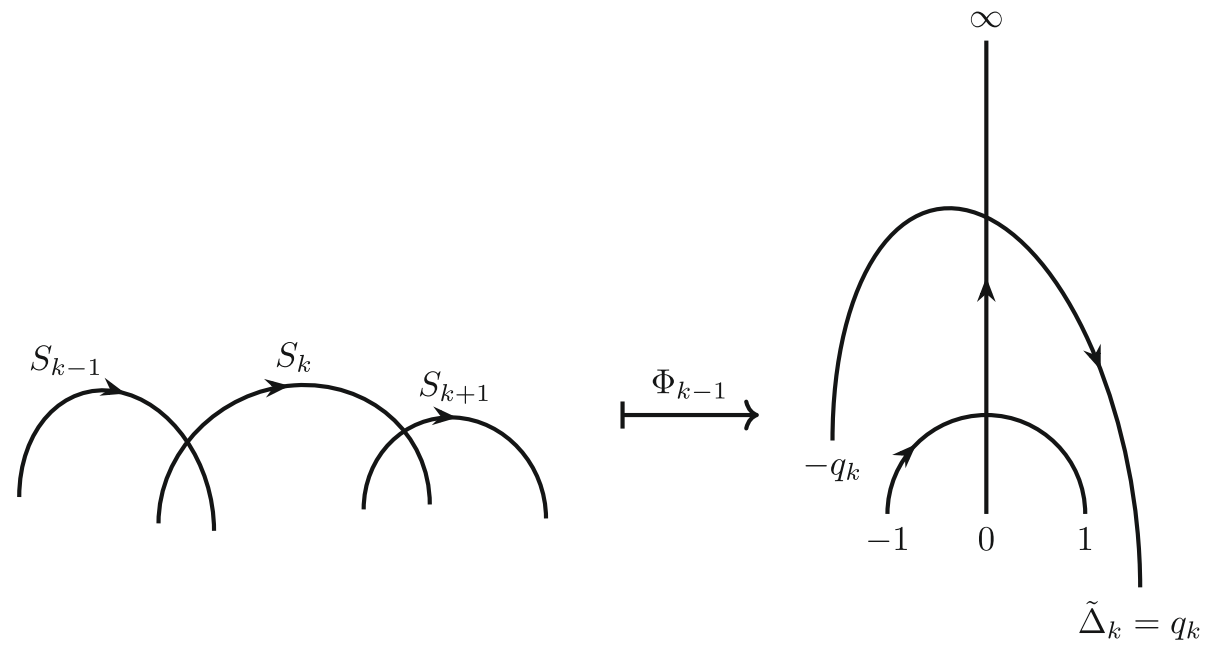

Fig. 3 Gauging by an isometry

of $(p-3)$-tuples of non-zero Clifford vectors. Denote by

$$
\begin{aligned}
& \operatorname{RAP}_{p}:=\left\{\left(S_{0}, \ldots, S_{p-1}\right) \text { non-degenerate right-angled polygon in } \boldsymbol{H}^{p-1}\right. \\
& \text { with } \left.S_{0}=(-1,1), S_{1}=(0, \infty)\right\}
\end{aligned}
$$

the set of non-degenerate right-angled polygons with $p$ sides. The calculation of the gauged double bridge cross ratios gives a map $\tilde{\Delta}: \operatorname{RAP}_{p} \rightarrow \mathcal{P}_{p}$. Denote the image of this map by $\mathcal{P}_{p}^{*}:=\tilde{\Delta}\left(\operatorname{RAP}_{p}\right) \subset \mathcal{P}_{p}$. This is the set of parameters which will yield a non-degenerate $\Pi_{p}$ in the construction below.

Theorem 1 The map $\tilde{\Delta}: \operatorname{RAP}_{p} \rightarrow \mathcal{P}_{p}^{*}$ is a bijection. The inverse map can be given as an explicit construction of a right-angled $p$-gon $\Pi_{p}$ from a tuple of $p-3$ parameters in $\mathcal{P}_{p}^{*}$.

\subsection{Proof of Theorem 1}

Bijectivity It is sufficient to prove the injectivity of $\tilde{\Delta}$ since it is surjective by definition. Note that in the standard configuration double bridge of Fig. 2, there is a one-to-one correspondence of Clifford vectors $x$ and geodesics $t=(-x, x)$ as given by Eq. (15). Now assume there are two $p$-gons $\Pi_{p}=\left(S_{0}, \ldots, S_{p-1}\right), \Pi_{p}^{\prime}=\left(S_{0}^{\prime}, \ldots, S_{p-1}^{\prime}\right) \in \mathrm{RAP}_{p}$ such that $\tilde{\Delta}\left(\Pi_{p}\right)=\tilde{\Delta}\left(\Pi_{p}^{\prime}\right)=\left(q_{1}, \ldots, q_{p-3}\right)$. By definition $S_{0}=S_{0}^{\prime}$ and $S_{1}=S_{1}^{\prime}$. By the above correspondence we also have $S_{2}=S_{2}^{\prime}$. Furthermore the maps $\phi_{1}, \ldots, \phi_{p-3}$ are the same for both $\Pi_{p}$ and $\Pi_{p}^{\prime}$ since these maps are defined by $q_{1}, \ldots, q_{p-3}$ as given in Eq. (16). Therefore the map $\Phi_{i}$ yields the same one-to-one correspondence between geodesics and Clifford vectors in both $p$-gons.

Construction of the polygon $\Pi_{p}$ The inverse map $\tilde{\Delta}^{-1}$ is given by the construction of a $\Pi_{p}$ from $p-3$ parameters $q_{1}, \ldots, q_{p-3} \in \mathbb{V}^{p-2}$.

Assume we are given $p-3$ parameters $\left(q_{1}, \ldots, q_{p-3}\right) \in \mathcal{P}_{p}^{*}$. 
Start The first two geodesics are fixed as $S_{0}=(-1,1)$ and $S_{1}=(0, \infty)$. Since this is the standard configuration double bridge considered above, we find $S_{2}=\left(-q_{1}, q_{1}\right)$ if we demand $\Delta\left(S_{0}, S_{1}, S_{2}\right)=q_{1}$.

The geodesic $S_{3}$ To find the endpoints of $S_{3}$, we benefit from the isometry $\phi_{1}^{-1}$ above which maps $(-1,1)$ to $(0, \infty)$ and $(0, \infty)$ to $S_{2}$. If $q_{2}$ was the cross ratio of a double bridge involving $(-1,1)$ and $(0, \infty)$, the third geodesic would be $\left(-q_{2}, q_{2}\right)$. Since $S_{3}$ is part of the double bridge starting with $(0, \infty)$ and $S_{2}, S_{3}$ can be found by applying $\phi_{1}^{-1}$ to $\left(-q_{2}, q_{2}\right)$, that is $S_{3}=\left(\phi_{1}^{-1}\left(-q_{2}\right), \phi_{1}^{-1}\left(q_{2}\right)\right)$.

The next geodesic in the general case The further procedure expands the previous idea. First we note that the next geodesic is given by the parameter $q_{3}$. The geodesic $S_{4}$ would then be the image of $\left(-q_{3}, q_{3}\right)$ under the isometry $\Phi_{2}^{-1}$ mapping $(-1,1)$ and $(0, \infty)$ to $S_{2}$ and $S_{3}$, respectively.

In general, assuming we have calculated the geodesics $S_{0}, \ldots, S_{k}$ for some $k$ with $2 \leq$ $k \leq p-3$, we can use $\Phi_{k-1}^{-1}$ in order to obtain $S_{k+1}=\left(\Phi_{k-1}^{-1}\left(-q_{k}\right), \Phi_{k-1}^{-1}\left(q_{k}\right)\right)$.

Existence of the last geodesic After using all the parameters $q_{1}, \ldots, q_{p-3}$, we have determined the geodesics $S_{0}, \ldots, S_{p-2}$. As a consequence of Proposition 2 the last common perpendicular between $S_{0}$ and $S_{p-2}$ exists and is unique as long as

$$
\Delta\left(S_{0}, S_{p-2}\right) \notin \mathbb{R}_{-} .
$$

This is ensured by the set $\mathcal{P}^{*} \subset \mathcal{P}$.

Remark 3 Since the Clifford vectors do not commute, one cannot directly compute the common perpendicular $S_{p-1}$ using the equations

$$
\Delta\left(S_{p-1}, S_{0}\right)=-1, \quad \Delta\left(S_{p-1}, S_{p-2}\right)=-1 .
$$

However, one can use an isometry to obtain a nice configuration where the terms in the equations above commute. Writing $S_{p-2}=(a, b)$, consider the isometry

$$
\psi: x \mapsto \alpha^{-1}(1+x)(1-x)^{-1} \alpha^{-1}
$$

where $\alpha:=\sqrt{-(1+a)(1-a)^{-1}}$. This isometry maps $S_{0}$ to $(0, \infty)$ and $S_{p-2}$ to $(1, c)$ where $c:=\alpha^{-1}(1+b)(1-b)^{-1} \alpha^{-1}$.

Hence, by Proposition 3

$$
S_{p-1}=\left(\psi^{-1}(-\sqrt{c}), \psi^{-1}(\sqrt{c})\right)
$$

modulo orientation where $\psi^{-1}$ is given by

$$
\psi^{-1}(x)=(\alpha x \alpha-1)(\alpha x \alpha+1)^{-1} .
$$

Remark 4 A major drawback is that we cannot explicitly describe $\mathcal{P}_{p}^{*}$. One can take a set of parameters $\left(q_{1}, \ldots, q_{p-3}\right) \in \mathcal{P}_{p}$, apply the above construction and afterwards check whether the created object actually is a non-degenerate right-angled $p$-gon.

If all the parameters $q_{i}$ have norm $\left|q_{i}\right|>1$ the proper orientation of the geodesics $S_{1}, \ldots, S_{p-3}$ is automatically guaranteed. So one needs to check the orientation of $S_{0}, S_{p-2}$ and $S_{p-1}$. This can be done by calculating the norm of the double bridge cross ratios with the respective geodesic as the central one. Since the norm of the cross ratio is invariant under isometry we do not have to use the gauged double bridge cross ratios at this point. If the 
orientation of $S_{p-1}$ is wrong, one can just invert it. If the orientation of $S_{p-2}$ is wrong, one needs to replace the parameter $q_{p-3}$ by $-q_{p-3}$ and the construction yields the same $\Pi_{p}$ just with the inverted orientation of $S_{p-2}$. If the orientation of $S_{0}$ is wrong, one can replace the parameter $q_{1}$ by $-q_{1}$. This introduces a factor $i$ to the left and to the right of the map $\phi_{1}^{-1}$, where $i$ is a root of -1 in the plane spanned by 1 and $q_{1}$; respectively $i=i_{1}$ if $q_{1}$ is real. Such a map is a rotation of $180^{\circ}$ in the plane spanned by 1 and $i$.

After some exemplary calculations, we conjecture that for $p=5$ the set

$$
\left\{\left(q_{1}, q_{2}\right) \in \mathcal{P}_{5} \mid \Re\left(q_{1}\right) \neq 0, q_{1} \not \perp q_{2}\right\}
$$

yields non-degenerate right-angled 5-gons up to orientation.

\section{Right-angled polygons with full span}

One natural question which arises when studying right-angled polygons is the question of the dimension of the resulting object. In this section we consider right-angled $p$-gons which have the highest possible dimension. This is the case if the $p$ intersection points are the vertices of a $(p-1)$-simplex. Thus the parameters will be taken from a $(p-2)$-dimensional Clifford vector space $\mathbb{V}^{p-2} \subset \mathcal{C}_{p-3}$.

\subsection{A necessary condition for the realisation of $(p-1)$-simplices}

If we want some set of parameters to yield a simplex, we need to pass to a new dimension with every new geodesic in the construction. This basic idea results in the following theorem:

Theorem 2 If the parameters $q_{1}, \ldots, q_{p-3} \in \mathcal{C}_{p-3}$ give rise to a right-angled polygon $\Pi_{p}$ whose intersection points are the vertices of a simplex, then the parameters together with 1 have to form a basis of the Clifford vectors according to $\left\langle 1, q_{1}, \ldots, q_{p-3}\right\rangle=\mathbb{V}^{p-2}$.

This theorem is a consequence of the following lemma:

Lemma 2 Let $\left(S_{0}, S_{1}, \ldots, S_{k}\right), k \geq 2$ be a finite sequence of geodesics in upper-half space $\boldsymbol{H}^{p-1}$ such that $S_{0}=(-1,1), S_{1}=(0, \infty)$ and $S_{i-1} \perp S_{i}$ for $i=1, \ldots, k$. Furthermore denote by $q_{i}:=\tilde{\Delta}\left(S_{i-1}, S_{i}, S_{i+1}\right)$ the gauged double bridge cross ratios of the respective double bridges for $i=1, \ldots, k-1$ and write $S_{i}=\left(S_{i}^{-}, S_{i}^{+}\right)$for all geodesics.

Then the linear subspace of $\mathbb{V}^{p-2}$ spanned by the endpoints of the geodesics is the same as the subspace spanned by $\left\{1, q_{0}, q_{1}, \ldots, q_{k-1}\right\}$. In symbols this means

$$
\left\langle S_{0}^{ \pm}, S_{2}^{ \pm}, S_{3}^{ \pm}, \ldots, S_{k}^{ \pm}\right\rangle=\left\langle 1, q_{1}, q_{2}, \ldots, q_{k-1}\right\rangle \text {. }
$$

The geodesic $S_{1}$ is left out since $\infty \notin \mathbb{V}^{p-2}$.

Proof We prove this by induction over $k$. For $k=2$ the lemma is plain, since $S_{2}=\left(-q_{1}, q_{1}\right)$.

Hence, we have to prove $\left\langle 1, q_{1}, q_{2}, \ldots, q_{k-1}, S_{k+1}^{ \pm}\right\rangle=\left\langle 1, q_{1}, q_{2}, \ldots, q_{k}\right\rangle$. We know that $S_{k+1}$ is given as the image of $\left(-q_{k}, q_{k}\right)$ under the isometry $\Phi_{k-1}^{-1}$. This isometry is given as a concatenation of the maps $\phi_{i}^{-1}: x \mapsto \sqrt{-q_{i}}(1+x)(1-x)^{-1} \sqrt{-q_{i}}, 1 \leq i \leq k-1$. If $q_{i} \notin \mathbb{R}, \phi_{i}^{-1}$ restricts to an isometry on $\boldsymbol{H}^{3}$ where the boundary is given as $\partial \boldsymbol{H}^{3}=$ $\left\langle 1, q_{i}\right\rangle \cup\{\infty\}$. Likewise, $\phi_{i}$ restricts to an isometry on $\boldsymbol{H}^{4}$ where the boundary is given as $\partial \boldsymbol{H}^{4}=\left\langle 1, q_{i}, q_{k}\right\rangle \cup\{\infty\}$. The case $q_{i} \in \mathbb{R}$ follows in the same manner, by yielding isometries leaving corresponding subspaces $\boldsymbol{H}^{2}$ and $\boldsymbol{H}^{3}$ invariant. Thus follows the statement. 
Fig. 4 Hyperbolic pentagon with right-angled cyclic edge path

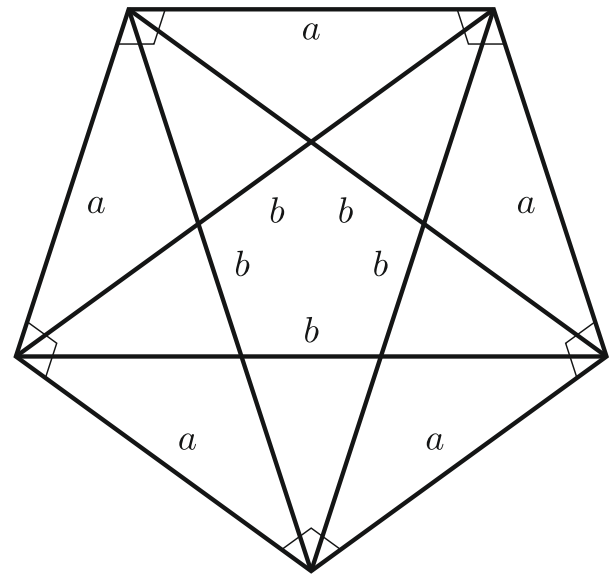

Notice that the theorem above does not give a sufficient condition. If the parameters $q_{i}$ are pairwise orthogonal to each other and pure Clifford vectors then the geodesics $S_{0}$ and $S_{p-2}$ will contribute sides of length 0 .

\subsection{Hyperbolic 4-simplices with an orthogonal cyclic edge path}

In the end, it would be nice to have an a priori condition on the parameters of at least some family of pentagons. Dekster and Wilker [6] proved a criterion for the existence of $n$ simplices with vertices $p_{1}, \ldots, p_{n+1}$ with given side and diagonal lengths $l_{i j}=\mathrm{d}\left(p_{i}, p_{j}\right)$, $1 \leq i<j \leq n+1$ in a Euclidean, spherical or hyperbolic space $X \in\left\{\boldsymbol{E}^{n}, \boldsymbol{S}^{n}, \boldsymbol{H}^{n}\right\}$. They call a symmetric $(n+1) \times(n+1)$-matrix $L=\left(l_{i j}\right)$ allowable if $l_{i i}=0$ and $l_{i j}>0$ for $i \neq j$. The matrix $L$ is called realisable in the space $X$ if there are $n+1$ points $p_{1}, \ldots, p_{n+1}$ in $X$ with the given distances $\mathrm{d}\left(p_{i}, p_{j}\right)=l_{i j}$. They gave a criterion for realisability in each of the three above cases. We are especially interested in the hyperbolic case.

Theorem 3 [6, Theorem 1 (hyperbolic case)] Let $L=\left(l_{i j}\right)$ be an allowable $(n+1) \times(n+1)$ matrix and let its entries be used to form the $(n \times n)$-matrix $S=\left(s_{i j}\right)$ where

$$
s_{i j}=\cosh l_{i, n+1} \cosh l_{j, n+1}-\cosh l_{i j} .
$$

Then $L$ is realisable if and only if the eigenvalues of $S$ are non-negative. If $L$ is realisable then the dimension of each realisation is equal to the rank of $S$.

Now we can easily treat the case of a hyperbolic pentagon having a cyclic edge path along which all sides have the same length (Fig. 4). With [6] we can get a criterion on the side lengths and due to symmetry it might be possible to find the corresponding orientations of the sides.

Lemma 3 A right-angled hyperbolic pentagon $\Pi_{5}=\left(S_{0}, \ldots, S_{4}\right)$ with all side lengths equal to $a>0$ is realisable as a 4-simplex if and only if $\cosh (a)<\gamma$, where $\gamma=\frac{1+\sqrt{5}}{2}$ denotes the golden ratio.

Proof By using hyperbolic trigonometry (see for example [12, Section 3.5]) we obtain the relation $\cosh (b)=\cosh ^{2}(a)$. We can now construct the two matrices $L$ and $S$ as in [6, Theorem 1]. We get 


$$
L=\left(\begin{array}{lllll}
0 & a & b & b & a \\
a & 0 & a & b & b \\
b & a & 0 & a & b \\
b & b & a & 0 & a \\
a & b & b & a & 0
\end{array}\right)
$$

Let us define $x:=\cosh (a)$. We then have

$$
S=\left(\begin{array}{cccc}
x^{2}-1 & x^{3}-x & x^{3}-x^{2} & 0 \\
x^{3}-x & x^{4}-1 & x^{4}-x & x^{3}-x^{2} \\
x^{3}-x^{2} & x^{4}-x & x^{4}-1 & x^{3}-x \\
0 & x^{3}-x^{2} & x^{3}-x & x^{2}-1
\end{array}\right) .
$$

By Dekster's and Wilker's Theorem, the matrix $L$ is realisable as a 4-simplex if and only if all the eigenvalues of $S$ are positive. This is true if and only if $S$ is positive definite. By Sylvester's criterion, it is enough to check that all the top left minors of $S$ have positive determinant:

$$
\begin{aligned}
\operatorname{det}_{1} & =x^{2}-1, \\
\operatorname{det}_{2} & =x^{4}-2 x^{2}+1=\left(x^{2}-1\right)^{2}=(x+1)^{2}(x-1)^{2}, \\
\operatorname{det}_{3} & =-x^{8}+2 x^{7}+x^{6}-2 x^{5}-2 x^{4}+3 x^{2}-1, \\
\operatorname{det}_{4}=\operatorname{det}(S) & =2 x^{10}-10 x^{9}+15 x^{8}-15 x^{6}+2 x^{5}+10 x^{4}-5 x^{2}+1 .
\end{aligned}
$$

Notice that $x>1$ since $a$ must be greater than 0 . Hence $\operatorname{det}_{1}$ and $\operatorname{det}_{2}$ are always greater than 0 . Furthermore, $\operatorname{det}_{3}$ is positive whenever $-1<x<\frac{1-\sqrt{5}}{2}$ or $1<x<\frac{1+\sqrt{5}}{2}$, hence only the latter has to be considered. The determinant of $S$ is positive everywhere except in $\frac{1-\sqrt{5}}{2}, 1, \frac{1+\sqrt{5}}{2}$, where it vanishes. Combining everything we obtain that $S$ is positive definite whenever $1<x<\gamma$, giving us the desired result.

Acknowledgements Both authors would like to thank their Ph.D. supervisor Prof. Ruth Kellerhals for her encouragement and valuable advice throughout the work on this paper and for her constant support.

\section{References}

1. Ahlfors, L.V.: Möbius transformations and Clifford numbers. In: Chavel, I., Farkas, H.M. (eds.) Differential geometry and complex analysis, pp. 65-73. Springer, Berlin (1985)

2. Ahlfors, L.V.: Möbius transformations in $\mathbb{R}^{n}$ expressed through $2 \times 2$ matrices of Clifford numbers. Complex Var. Elliptic Equ. 5(2-4), 215-224 (1986)

3. Beardon, A.F.: The Geometry of Discrete Groups, vol. 91. Springer, Berlin (2012)

4. Cao, C., Waterman, P.L.: Conjugacy invariants of Möbius groups. In: Duren, P., Heinonen, J., Osgood, B., Palka, B. (eds.) Quasiconformal mappings and analysis, pp. 109-139. Springer, New York (1998)

5. Costa, A.F., Martínez, E.: On hyperbolic right-angled polygons. Geom. Dedicata 58(3), 313-326 (1995). https://doi.org/10.1007/BF01263459

6. Dekster, B.V., Wilker, J.B.: Simplexes in spaces of constant curvature. Geom. Dedicata 38(1), 1-12 (1991). https://doi.org/10.1007/BF00147732

7. Delgove, F., Retailleau, N.: Sur la classification des hexagones hyperboliques à angles droits en dimension 5. Ann. Fac. Sci. Toulouse Math. 23(5), 1049-1061 (2014). https://doi.org/10.5802/afst.1435

8. Fathi, A., Laudenbach, F., Poénaru, V.: Travaux de Thurston sur les surfaces: Séminaire Orsay. Astérisque 66-67 (1979)

9. Fenchel, W.: Elementary Geometry in Hyperbolic Space, De Gruyter Studies in Mathematics, vol. 11. Walter de Gruyter \& Co., Berlin (1989). https://doi.org/10.1515/9783110849455

10. Parizet, J.: Quaternions et géométrie. http://www.math.unicaen.fr/lmno/semana/documents/parizet/ Quaternions_A.pdf (2006). Accessed 11 July 2017 
11. Parker, J.: Hyperbolic spaces. http://maths.dur.ac.uk/ dma0jrp/img/HSjyvaskyla.pdf (2007). Accessed: 11 July 2017

12. Ratcliffe, J.G.: Foundations of Hyperbolic Manifolds. Graduate Texts in Mathematics, vol. 149, 2nd edn. Springer, New York (2006). https://doi.org/10.1007/978-1-4757-4013-4

13. Vahlen, K.T.: Über bewegungen und complexe zahlen. Math. Ann. 55(4), 585-593 (1902)

14. Waterman, P.L.: Möbius transformations in several dimensions. Adv. Math. 101(1), 87-113(1993). https:// doi.org/10.1006/aima.1993.1043

15. Wilker, J.B.: Inversive geometry. In: Davis, C., Grünbaum, B., Sherk, F.A. (eds.) The geometric vein, pp. 379-442. Springer, Berlin (1981) 